\title{
Forecasting population trend from the scaling pattern of occupancy
}

\author{
Cang Hui* \\ Centre for Invasion Biology, Department of Botany and Zoology, Stellenbosch University, Private Bag X1, Matieland 7602, South Africa
}

\section{A R T I C L E I N F O}

\section{Article history:}

Received 19 July 2010

Received in revised form 22 October 2010

Accepted 25 October 2010

Available online 22 November 2010

\section{Keywords:}

Spatial scale

Pair approximation

Spatial autocorrelation

Area of occupancy

Population dynamics

Occupancy

Scaling

Allee effect

Biodiversity conservation

\begin{abstract}
A B S T R A C T
Forecasting the temporal trend of a focal species, its range expansion or retraction, provides crucial information regarding population viability. To this end, we require the accumulation of temporal records which is evidently time consuming. Progress in spatial data capturing has enabled rapid and accurate assessment of species distribution across large scales. Therefore, it would be appealing to infer the temporal trends of populations from the spatial structure of their distributions. Based on a combination of models from the fields of range dynamics, occupancy scaling and spatial autocorrelation, here I present a model for forecasting the population trend solely from its spatial distribution. Numerical tests using cellular automata confirm a positive correlation, as inferred from the model, between the temporal change in species range sizes and the exponent of the power-law scaling pattern of occupancy. The model is thus recommended for rapid estimation of species range dynamics from a single snapshot of its current distribution. Further applications in biodiversity conservation could provide a swift risk assessment, especially, for endangered and invasive species.
\end{abstract}

(c) 2010 Elsevier B.V. All rights reserved.

\section{Introduction}

Conservation has entered a new era where the spatial information regarding species distribution is more readily obtainable than temporal records on population dynamics. Consequently, spatial techniques (e.g. the remote sensing and geographical information system) and methods (e.g. site occupancy models and species niche modelling) for accurately estimating species range flourish (MacKenzie et al., 2003; McPherson et al., 2006; Gaston and Fuller, 2009), resulting in a spatial underpinning of current conservation planning (e.g. Roura-Pascual et al., 2010), e.g. the endorsement of the area of occupancy by the IUCN as one key criteria for species conservation status (Gardenfors et al., 1999). Given the current alarming rate of extinction due to climate change and biotic homogenization via globalization (e.g. Gaertner et al., 2009; Keith et al., 2009; Olden et al., 2010), it would be especially useful if we could provide a rapid assessment of whether some of the endangered species are declining, or whether the range of an invasive species is expanding.

Species range is not static, but dynamic (e.g. Hengeveld, 1989; Hui and Li, 2003). As such, the rate of expansion and retraction (i.e. the distributional trend) encompasses crucial information for pop-

\footnotetext{
* Corresponding author.

E-mail address: chui@sun.ac.za
}

ulation viability (Beissinger and Westphal, 1998; Hui, 2007). For instance, species ranges have been predicted to shift poleward in response to current climate change (Thomas et al., 2004), including the expansion of the high-latitude range margin and the erorsion of the low-latitude range margin (Murphy et al., 2010). However, even though this dynamic feature of species range has been emphasized in global change biology, it receives much less attention in conservation biology, not due to omission or ignorance, but rather because collecting long-term sampling data is costly and time-consuming, and is often unfeasible for most species of interest. Furthermore, to forecast future distributional trends, it is necessary to accumulate sufficient historical records to allow robust projection. Efficient conservation management and planning cannot afford such a waitand-see approach. Conservation management would stand in a better position if it were possible to convert the spatial data of a species distribution into the knowledge of its temporal trend. However, forecasting distributional trends from a snapshot of the current distribution seems an ideal beyond the reach of current models.

Changes of population sizes across time and space are entangled. For instance, space-for-time substitution has been applied in studies on plant succession after disturbance (Cooper, 1923; Crocker and Major, 1955) and the impact of biological invasions on native ecosystems (Fukami and Wardle, 2005), yet both have been heavily criticized because species spatial distribution and temporal dynamics are often governed by different ecological processes (Tokarska-Guzik et al., 2008). As a rule of thumb, the space-for- 
time substitution is to be avoided in ecology. However, these examples do indicate the potential correlation between changes in population size (or range size) across time and space. By comparing the atlases of British butterflies calibrated from different periods (1970-1982 and 1995-1999), Wilson et al. (2004) discovered a strong correlation between the change in species range sizes and the exponent of the power-law scaling pattern of species distributions (namely, the area-of-occupancy, AOO). Currently, no theoretical models are available for this strong correlation. Here, using a series of models from different fields, I provide a theoretical understanding of this correlation and further discuss the possibility of using this correlation to forecast population trends.

\section{Models}

Species range can be quantified as occupancy, i.e. the proportion of occupied cells on a grid-based atlas (Gaston and Fuller, 2009), whose dynamics can be depicted by Levins' patch occupancy model (Hanski, 1998),

$$
\frac{d p_{+}}{d t}=c \cdot p_{+}\left(1-p_{+}\right)-e \cdot p_{+}
$$

where $p_{+}$indicates species occupancy, $c$ and $e$ the rate of colonization and extinction. Ecological processes (e.g. colonization) are distance-limited, forming the pattern of spatial autocorrelation (Fortin and Dale, 2005; Hui et al., 2010) where the spatial pattern is constantly changing in response to the demographic dynamics and environmental changes. This model is essentially similar to a logistic equation and reflects negative density dependence in the population dynamics (Hanski, 1999). Other mechanisms of positive density dependence are also common in nature (e.g. the Allee effect; Berec et al., 2007). However, considering multiple density-dependent mechanisms inevitably makes the model too complicated to be analytically analyzed (e.g. Hui and Li, 2004). We here only consider the simple version as in Eq. (1), and leave the effect of other density-dependent mechanisms in Section 4.

The dynamics of the spatial pattern can be depicted by a simple spatially explicit model (Sato and Iwasa, 2000; Hui and Li, 2004),

$\frac{d p_{+}}{d t}=c \cdot p_{+}\left(1-q_{+/+}\right)-e \cdot p_{+}$

where $q_{+++}\left(\max \left\{0,2-1 / p_{+}\right\}<q_{+/+}<1\right)$ denotes the conditional probability of an occupied cell being adjacent to a known occupied cell. Eq. (2) depicts the temporal dynamics of species occupancy balanced by local colonization from adjacent populations and extinctions within cells. The spatial autocorrelation of species distribution can be measured by the joint-count statistics, $q_{+l_{+}} / p_{+}$ with a value of one indicating randomness and above one a positive spatial autocorrelation (Hui et al., 2006). By assuming that migrants are only from four most adjacent cells (i.e. von Neumann neighborhood) and also that the statuses of non-adjacent cells are independent from one another (i.e. pair approximation), we can derive the equilibrium of the above system (Hui and $\mathrm{Li}$, 2004): $\hat{p}_{+}=1-3 e /(3 c-e)$ and $\hat{q}_{+/+}=1-e / c$. Evidently, we have $\hat{q}_{+/+} / \hat{p}_{+}>1$, suggesting a positive spatial autocorrelation when the population reaches its equilibrium.

Occupancy is scale dependent (Hartley and Kunin, 2003; Gaston and Fuller, 2009; Hui and McGeoch, 2008), and this scale dependence suggests a percolation process of species distribution with the increase of cell size (Hui and McGeoch, 2007a). The AOO, i.e. the scaling pattern of occupancy, depicts how species occupancy changes across scales and has been reported to follow a power law with cell size (Kunin, 1998),

$p_{+} \sim a^{b}$, where $a$ indicates the cell size and $b$ the exponent of the AOO. A power-law form of the AOO implies a self-similar (fractal) structure of species distribution (Harte et al., 1999; Hartley and Kunin, 2003; Hui and McGeoch, 2007b). Even though this scale-invariant pattern does not hold across all scales (Lennon et al., 2007; Hui and McGeoch, 2007c), this power law function, nonetheless, does remain at a certain range of scales (Hartley and Kunin, 2003; Hui, 2009). The AOO exponent, thus, can be considered as a specific measure of species aggregation (Hui et al., 2010). In practice, the exponent can be estimated as:

$b=\frac{\ln \left(p_{+}^{*} / p_{+}\right)}{\ln 4}$

where $p_{+}^{*}$ is the new occupancy at a coarser scale after joining four neighboring cells together and can be estimated by a Bayesian model from $p_{+}$and $q_{+/+}$alone (Hui, 2009; Hui et al., 2006),

$p_{+}^{*}=1-\frac{\nabla^{4}}{\Delta}$,

where $\nabla=1-p_{+}-\left(1-q_{+++}\right) p_{+}$and $\Delta=\left(1-p_{+}\right)\left(1-p_{+}^{2}\left[2 q_{+/+}-\right.\right.$ $\left.3]+p_{+}\left[q_{+/+}^{2}-3\right]\right)$. This provides a theoretical model for examining the relationship between the snapshot of a species distribution, quantified by the AOO exponent $(b)$ and the distributional trend $\left(d p_{+} / d t\right)$

To verify the model prediction, we built a cellular automaton of metapopulations. Cellular automata have been widely used in ecological modelling and conservation management (e.g. RouraPascual et al., 2009; Craig, 2010) and are a robust tool for analyzing spatial pattern formation and spatiotemporal dynamics (Hui and Yue, 2005; Su et al., 2009). In this cellular automaton, we assigned the probability of an occupied cell becoming empty in the next time step with $\operatorname{Pr}(1 \rightarrow 0)=e$ and the probability of an empty cell being colonized with $\operatorname{Pr}(0 \rightarrow 1)=(c / 4) \sum_{i=1}^{4} p_{i}$, where $p_{i}$ is the status of one of the four adjacent cells, either occupied $\left(p_{i}=1\right)$ or empty $\left(p_{i}=0\right)$. The cellular automaton was run on a $40 \times 40$ lattice, and the occupancy $\left(p_{+}\right)$was measured as the proportion of occupied cells. To calculate the occupancy at a coarser scale, four adjacent cells $(\{2 i-1,2 j-1\},\{2 i, 2 j-1\},\{2 i-1,2 j\},\{2 i, 2 j\})$ were then combined into a bigger cell $\{i, j\}$ (where $i, j=1,2, \ldots, 20$ ), forming a $20 \times 20$ lattice.

\section{Numerical evaluation}

First, to investigate the model behaviour, we uniformly chose all feasible combinations of the occupancy $\left(p_{+}\right)$and the conditional probability $\left(q_{+++}\right)$, i.e. $0 \leq p_{+} \leq 1$ and $\max \left\{0,1-2 / p_{+}\right\} \leq q_{+++} \leq 1$, and found that the distributional trend was positively correlated with the AOO exponent (Fig. $1 \mathrm{~A}, r=0.76, p<0.01$ ). The rates of colonization and extinction, being the only two parameters in the model, had an obvious impact on the relationship between the AOO exponent and the distributional trend. The slope of the linear regression between the AOO exponent and the distribution trend ascended in general with the increase of the extinction-to-colonization ratio $(e / c)$ and was upper bounded by a straight line, slope $=0.5 e / c+0.14$ (Fig. 1B).

Second, simulations using the cellular automaton (Fig. 2) revealed that the AOO exponent declined in an expanding population (red dots in Fig. 1C), whereas it increased in a retracting population (blue dots in Fig. 1C). It is worth noting that a few points have fallen outside of the model prediction. This suggests that there is a certain level of deviation between the estimates of the AOO exponent from the Bayesian estimation (Eq. 5) and those exponents directly observed from the simulation. The AOO exponent in both expanding and retracting populations was approaching an equilibrium that can be completely determined by the extinction- 

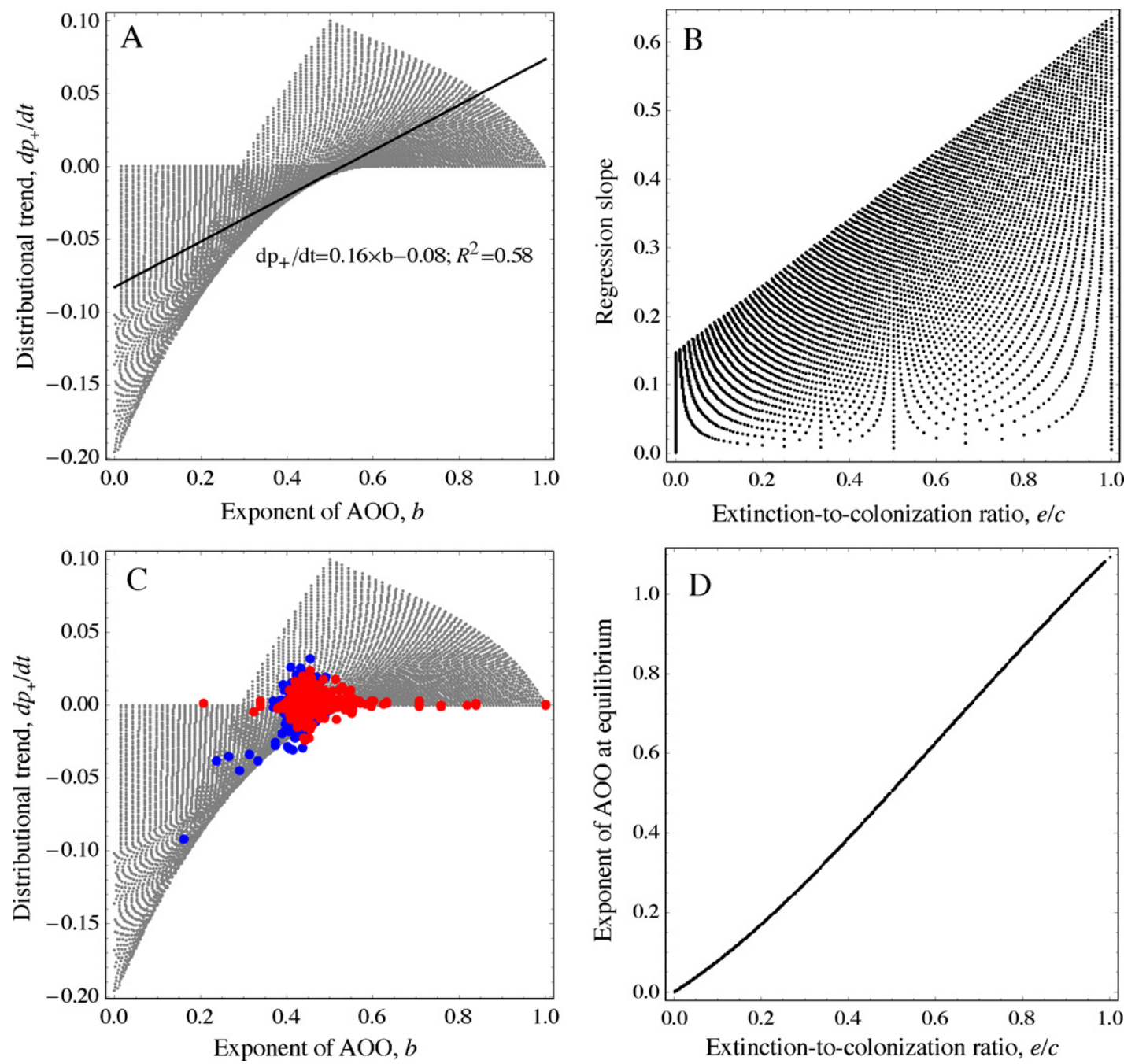

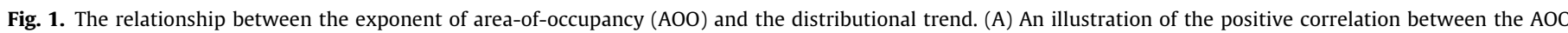

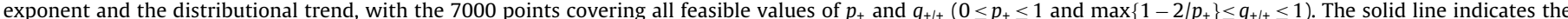

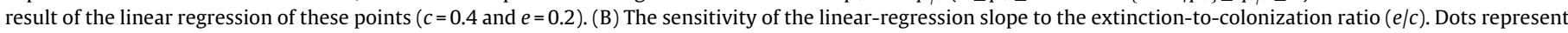

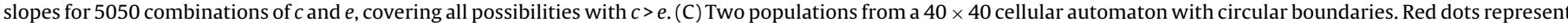

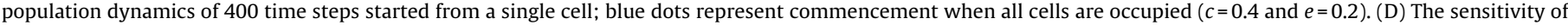

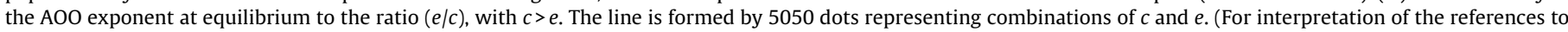
colour in this figure legend, the reader is referred to the web version of the article.)
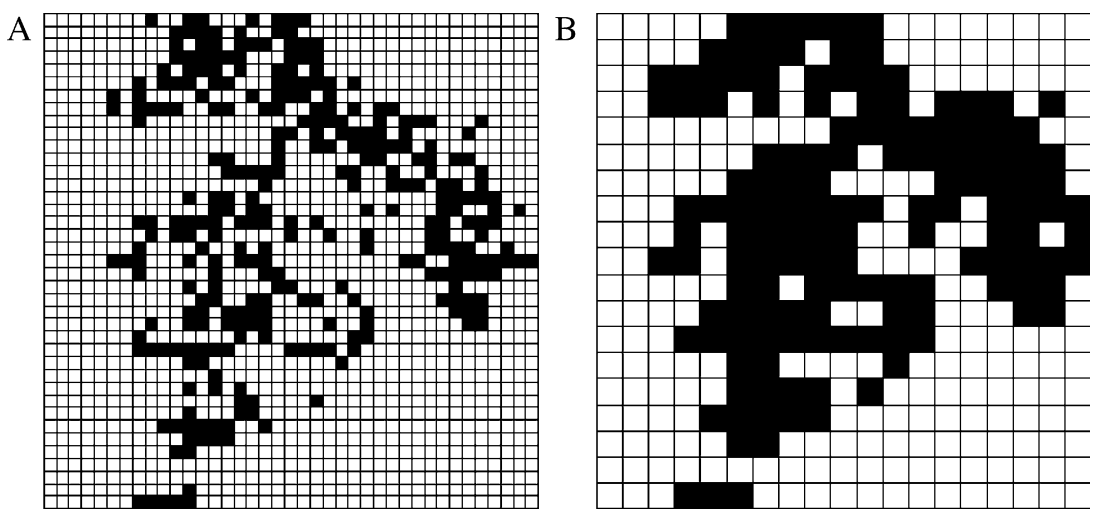

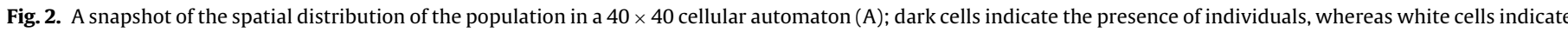

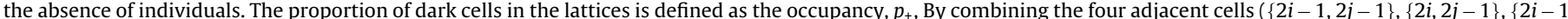

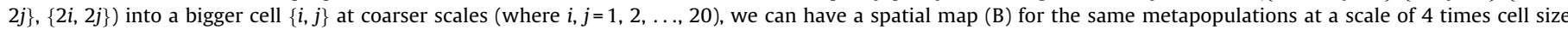

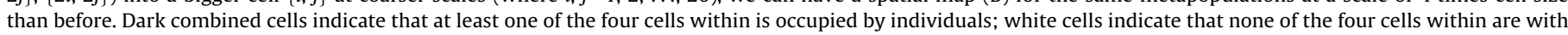
individuals. The proportion of dark cells gives the occupancy at a coarse scale in Eq. (4), $p_{+}^{*}$, which can be estimated by the Bayesian model (Eq. (5)). 
to-colonization ratio (Fig. 1D), i.e. the spatial structure of species distribution (b) can be fully determined by population's temporal rates of change ( $e$ and $c$ ). This is because the colonization and extinction rates determine the equilibrium of the system (i.e. $\hat{p}_{+}$ and $\left.\hat{q}_{+/+}\right)$, and thus determine the AOO exponent. Overall, there existed a strong positive correlation between the AOO exponent and species distributional trend. Forecasting population trend from the scaling pattern of occupancy was thereby made possible.

\section{Discussion}

These results suggest a positive correlation between the AOO exponent and the distributional trend, $d p_{+} / d t \sim+b$. A steeper AOO entails that the population is more likely to expand in the near future, whereas a shallower AOO suggests a retracting population. Wilson et al. (2004) discover, for British butterflies (and also for other taxa in Europe), a positive relationship between the change of range size and the occupancy, $d p_{+} / d t=0.18 p_{+}-0.97$, concurring with Borregaard and Rahbek's (2006) observations that widespread species are more likely to expand their range than rare species. It implies a threshold of species occupancy $\left(p_{+}^{\prime}=0.97 / 0.18\right)$ where rare species decline (if $p_{+}<p_{+}^{\prime}$, then $d p_{+} / d t<0$ ) and common species increase (if $p_{+}>p_{+}^{\prime}$, then $d p_{+} / d t>0$ ). This is a clear indication of the Allee effect, a mechanism of the positive density dependence when the population size is small (Berec et al., 2007; Chen and Hui, 2009). Wilson et al. (2004) further discover a positive relationship between the fractal dimension $(D)$ and the change of range size, implying a negative correlation between the distributional trend and the AOO exponent, $d p_{+} / d t=-0.96 \cdot b+0.17$ ( $b=1-D / 2$; Kunin, 1998). This seeming contradiction to our results is due to the threshold phenomenon caused by the positive densitydependent Allee effect, whereas here we only demonstrated the correlation for the general negative density-dependent population dynamics. Therefore, we can conclude that: (i) the future change in species range size is correlated with the scaling pattern of occupancy; (ii) the exact form of this correlation is dependent on the density-dependent mechanisms.

Ecologists need not wait to see the trend of a focal species; the current species distribution, its scaling pattern of occupancy per se, has already provided clues to its future. Measuring the scaling pattern of species distribution thus provides a swift tool for assessing and forecasting the population dynamics of species (Wilson et al., 2004). This enables the inclusion of population trend as an indicator for species conservation status. Two kinds of species specifically require constant monitoring and rapid assessment of their population trends - endangered and invasive species. For instance, historic records for those well-known invasive species are quite available, whilst rare for endangered species. This could mean that the model is more relevant to endangered species than to invasive species. Besides conserving the remaining habitat and individuals for endangered species, prioritization should be given to the strategies of habitat management that can forecast an increase of population size. In the same way, control and eradication plans for invasive species should be assessed a prior to ensure a negative population trend for mitigating its potential impact on the recipient environment. Using this model in biodiversity conservation to forecast population trends is possible and warrants further attention.

Future research of the model lies in three directions. Firstly, it is necessary to examine the correlation between the distributional trend and the AOO exponent under different and more realistic density-dependent mechanisms (e.g. under the Allee effect and overcrowding effect). Secondly, we here only used the joint-count statistics for quantifying the spatial autocorrelation and a power law for the occupancy scaling. Measures of aggregated species dis- tribution abound (e.g. Fortin and Dale, 2005; Hui et al., 2010), such as the fractal dimension and other indices that depict the distance decay of similarity in species distribution. These measures of species nonrandom distribution (and spatial heterogeneity) are usually classified into three groups based on their conceptual relationships (Wiens, 2000; Dungan et al., 2002), whereas four groups have been identified based on their scaling patterns (Hui et al., 2010). It would be interesting to distinguish measures which are correlated with population dynamics from those which are not. Finally, range (occupancy) dynamics is not equal to the dynamics of population size (abundance). It is possible that individuals cluster together to enhance fitness (e.g. insect swarm) and thus exhibit small range with increasing density. Future research on the synchronicity of species range dynamics and its demographic dynamics (e.g. Borregaard and Rahbek, 2006) could be fruitful for biodiversity conservation, and the species occupancy-abundance relationship provides a starting point for demonstrating the relationship between the dynamics of species range size and population density (Borregaard and Rahbek, 2010; Gaston and Blackburn, 2000; Hui et al., 2009).

\section{Acknowledgments}

I am grateful to Brian Fath, B. Laniewski and two anonymous reviewers for constructive comments. This work is supported by the NRF Blue Skies Programme, the Subcommittee B fund at Stellenbosch University and the DST-NRF Centre of Excellence for Invasion Biology.

\section{References}

Beissinger, S.R., Westphal, M.I., 1998. On the use of demographic models of population viability in endangered species management. Journal of Wildlife Management 62, 821-841.

Berec, L., Angulo, E., Courchamp, F., 2007. Multiple Allee effects and population management. Trends in Ecology and Evolution 22, 185-191.

Borregaard, M.K., Rahbek, C., 2006. Prevalence of intraspecific relationships between range size and abundance in Danish birds. Diversity and Distributions 12 , $417-422$.

Borregaard, M.K., Rahbek, C., 2010. Causality of the relationship between geographic distribution and species abundance. Quarterly Review of Biology 85, 3-25.

Chen, L.-L., Hui, C., 2009. Habitat destruction and the extinction debt revisited: the Allee effect. Mathematical Biosciences 221, 26-32.

Cooper, W.S., 1923. The recent ecological history of Glacier Bay, Alaska. Ecology 4, 223-246.

Craig, P.D., 2010. Imposed and inherent scales in cellular automata models of habitat. Ecological Modelling 221, 2425-2434.

Crocker, R.L., Major, J., 1955. Soil development in relation to vegetation and surface age at Glacier Bay, Alaska. Journal of Ecology 43, 427-448.

Dungan, J.L., Perry, J.N., Dale, M.R.T., Legendre, P., Citron-Pousty, S., Fortin, M.-J., Jakomulska, A., Miriti, M., Rosenberg, M.S., 2002. A balanced view of scale in spatial statistical analysis. Ecography 25, 626-640.

Fortin, M.J., Dale, M.R.T., 2005. Spatial Analysis: A Guide for Ecologists. Cambridge University Press, Cambridge.

Fukami, T., Wardle, D.A., 2005. Long-term ecological dynamics: reciprocal insights from natural and anthropogenic gradients. Proceeding of the Royal Society B: Biological Sciences 272, 2105-2115.

Gaertner, M., den Breeyen, A., Hui, C., Richardson, D.M., 2009. Impacts of alien plant invasion on species richness in Mediterranean-type ecosystems: a metaanalysis. Progress in Physical Geography 33, 319-338.

Gardenfors, U., Rodriguez, J.P., Hilton-Taylor, C., Hyslop, C., Mace, G., Molur, S., Poss, S., 1999. Draft guidelines for the application of IUCN Red List Criteria at national and regional levels. Species 31/32, 58-70.

Gaston, K.J., Blackburn, T.M., 2000. Pattern and Process in Macroecology. Blackwell Science, Oxford.

Gaston, K.J., Fuller, R.A., 2009. The sizes of species' geographic ranges. Journal of Applied Ecology 46, 1-9.

Hanski, I., 1998. Metapopulation dynamics. Nature 396, 41-49.

Hanski, I., 1999. Metapopulation Ecology. Oxford University Press, Oxford.

Harte, J., Kinzig, A., Green, J., 1999. Self-similarity in the distribution and abundance of species. Science $284,334-336$.

Hartley, S., Kunin, W.E., 2003. Scale dependency of rarity, extinction risk, and conservation priority. Conservation Biology 17, 1559-1570.

Hengeveld, R., 1989. Dynamics of Biological Invasions. Chapman and Hall, London.

Hui, C., 2007. Negative correlation between dynamical complexity and metapopulation persistence: a reply. Ecological Modelling 200, 271-272. 
Hui, C., 2009. On the scaling patterns of species spatial distribution and association. Journal of Theoretical Biology 261, 481-487.

Hui, C., Li, Z.Z., 2003. Dynamical complexity and metapopulation persistence. Ecological Modelling 164, 201-209.

Hui, C., Li, Z.Z., 2004. Distribution patterns of metapopulation determined by Allee effects. Population Ecology 46, 55-63.

Hui, C., McGeoch, M.A., 2007a. Capturing the "droopy-tail" in the occupancyabundance relationship. Ecoscience 14, 103-108.

Hui, C., McGeoch, M.A., 2007b. A self-similarity model for the occupancy frequency distribution. Theoretical Population Biology 71, 61-70.

Hui, C., McGeoch, M.A., 2007c. Modeling species distributions by breaking the assumption of self-similarity. Oikos 116, 2097-2107.

Hui, C., McGeoch, M.A., 2008. Does the self-similar species distribution model lead to unrealistic predictions? Ecology 89, 2946-2952.

Hui, C., McGeoch, M.A., Reyers, B., le Roux, P.C., Greve, M., Chown, S.L., 2009. Extrapolating population size from the occupancy-abundance relationship and the scaling pattern of occupancy. Ecological Applications 19, 2038-2048.

Hui, C., McGeoch, M.A., Warren, M., 2006. A spatially explicit approach to estimating species occupancy and spatial correlation. Journal of Animal Ecology 75, 140-147.

Hui, C., Veldtman, R., McGeoch, M.A., 2010. Measures, perceptions and scaling patterns of aggregated species distributions. Ecography 33, 95-102.

Hui, C., Yue, D., 2005. Niche construction and polymorphism maintenance in metapopulations. Ecological Research 20,115-119.

Keith, S.A., Newton, A.C., Morecroft, M.D., Bealey, C.E., Bullock, J.M., 2009. Taxonomic homogenization of woodland plant communities over 70 years. Proceeding of the Royal Society B: Biological Sciences 276, 3539-3544.

Kunin, W.E., 1998. Extrapolating species abundance across spatial scales. Science $281,1513-1515$.

Lennon, J.J., Kunin, W.E., Hartley, S., Gaston, K.J., 2007. Species distribution patterns, diversity scaling and testing for fractals in southern African birds. In: Storch, D., Marquet, P.A., Brown, J.H. (Eds.), Scaling Biodiversity. Cambridge University Press, Cambridge, pp. 51-76.

MacKenzie, D.I., Nichols, J.D., Hines, J.E., Knutson, M.G., Franklin, A.D., 2003. Estimating site occupancy, colonization and local extinction probabilities when a species is not detected with certainty. Ecology 84, 2200-2207.
McPherson, J.M., Jetz, W., Rogers, D.J., 2006. Using coarse-grained occurrence data to predict species distributions at finer spatial resolutions - possiblities and limitations. Ecological Modelling 192, 499-522.

Murphy, H.T., van der Wal, J., Lovett-Doust, J., 2010. Signiatures of range expansion and eroison in eastern North American trees. Ecology Letters 13, 1233-1244.

Olden, J.D., Kennard, M.J., Leprieur, F., Tedesco, P.A., Winemiller, K.O., GarciaBerthou, E., 2010. Conservation biogeography of freshwater fishes: recent progress and future challenges. Diversity and Distributions 16, 496-513.

Roura-Pascual, N., Bas, J.M., Thuiller, W., Hui, C., Krug, R.M., Brotons, L., 2009. From introduction to equilibirium: reconstructing the invasive pathways of the Argentine ant in a Mediterranean region. Global Change Biology 15, 2101-2115.

Roura-Pascual, N., Krug, R.M., Richardson, D.M., Hui, C., 2010. Spatially-explicit sensitivity analysis for conservation management: exploring the influence of decisions in invasive alien plant management. Diversity and Distributions 16, 426-438.

Sato, K., Iwasa, Y., 2000. Pair approximations for lattice-based ecological models. In: Dieckmann, U., Law, R., Metz, J.A.J. (Eds.), The Geometry of Ecological Interactions: Simplifying Spatial Complexity. Cambridge University Press, Cambridge, pp. 341-358.

Su, M., Hui, C., Zhang, Y.Y., Li, Z.Z., 2009. How does the spatial structure of habitat loss affect the eco-epidemic dynamics? Ecological Modelling 220, 51-59.

Thomas, C.D., Cameron, A., Green, R.E., Bakkenes, M., Beaumont, L.J., Collingham, Y.C. Erasmus, B.F.N., de Siqueira, M.F., Grainger, A., Hannah, L., Hughes, L., Huntley, B., van Jaarsveld, A.S., Midgley, G.F., Miles, L., Ortega-Huerta, M.A., Peterson, A.T., Philips, O.L., Williams, S.E., 2004. Extinction risk from climate change. Nature $427,145-148$.

Tokarska-Guzik, B., Brock, J., Brundu, G., Child, L., Daehler, C., Pysek, P., 2008. Plant Invasions: Human Perception, Ecological Impacts and Managment. Backhuys Publishers, Leiden, The Netherlands.

Wiens, J.A., 2000. Ecological heterogeneity: ontogeny of concepts and approaches In: Hutchings, M.J., John, E.A., Stewart, A.J.A. (Eds.), The Ecological Consequences of Environmental Heterogeneity. Blackwell, Oxford, pp. 9-31.

Wilson, R.J., Thomas, C.D., Fox, R., Roy, D.B., Kunin, W.E., 2004. Spatial patterns in species distributions reveal biodiversity change. Nature 432, 393-396. 